\title{
A Full-Wave Analysis of Microstrip Lines by Variational Conformal Mapping Technique
}

\author{
CHIN SHIH, RUEY-BEEI WU, SHYH-KANG JENG, MEMBER, IEEE, \\ AND CHUN HSIUNG CHEN
}

\begin{abstract}
A novel full-wave analysis of microstrip lines is presented. Wheeler's mapping, which is useful in the quasi-TEM analysis of microstrip lines, is combined with the full-wave variational formulation to facilitate a finite element solution. This desirable mapping not only transforms the problem domain into a finite region, but also overcomes the field singularity on the strip edge. Compared with other known techniques, the present method makes fewer assumptions, and is more rigorous as long as the strip thickness is negligible. Numerical results for the frequency dependence of effective dielectric constant, the characteristic impedance, and both longitudinal and transverse current distributions on the strip are also included.
\end{abstract}

\section{INTRODUCTION}

$\mathbf{M}$ ICROSTRIP HAS BECOME the most popular and important element in microwave integrated circuits (MIC's) and microwave networks in the band $1 \sim 30 \mathrm{GHz}$ [1]-[4]. In earlier work microstrip was regarded as a quasiTEM mode transmission line [5]-[8]. Hence the resultant parameters, such as the effective dielectric constant and characteristic impedance, are independent of frequency.

In order to examine the dispersion characteristics, several methods [9]-[19] have been developed. Most of them first derived an integral equation in terms of current or charge on the strip, then made an approximation for the current or charge distributions by physical intuition to carry out numerical analysis. Daly tried the finite element method [17]; however, he solved only the closed microstrip line, and did not take good account of the boundary and edge conditions on the strip. Others developed dispersion models [18], [19] through approximating the microstrip lines as suitable waveguides, which are easier to analyze.

Recently, some authors [20]-[22] have made efforts to solve numerically current and charge distributions on the strip by Green's function techniques. The approximate close forms of current distributions which are independent of frequency and dielectric constant are obtained from the complicated quasi-static solutions.

Manuscript received May 12, 1987; revised September 29, 1987. This work was supported by the National Science Council, Republic of China, under Grant NSC 76-0404-E002-13.

The authors are with the Department of Electrical Engineering, National Taiwan University, Taipei, Taiwan, R.O.C. From August 1986 to July 1987, C. H. Chen was on sabbatical leave at the Department of Electrical Engineering, University of Houston, Houston, TX 77004.
This paper proposes a rigorous method based on conformal mapping and variational reaction theory [23] to improve the numerical accuracy. This idea has been used successfully in handling various open dielectric waveguides [23], such as image guides, strip guides, and channel guides. However, due to the existence of field singularities on the edge of the open microstrip line, microstrip line problems would be more difficult to solve than dielectric waveguide problems. In this article, Wheeler's mapping [7], [8] will be used to take care of these singularities. Thus, the current distributions on the strip can be obtained with minimal approximations. Also computed are the effective dielectric constant and characteristic impedance.

\section{VARIATIONAL ReACTION THEORY}

This section summarizes the basic notion of the variational reaction theory (VRT), which constructs a variational formulation to EM field problems. We shall consider a trial system in addition to the solution system (the original system) and find a functional $I$ of the fields in the trial system such that its first variation $\delta I$ vanishes at the true fields of the solution system [24].

First consider the trial fields $(\bar{E}, \bar{H})$ supported by the trial sources $(\bar{J}, \bar{M})$ :

$$
\begin{aligned}
& \bar{J}=\nabla \times \bar{H}-j \omega \epsilon \bar{E} \\
& \bar{M}=-\nabla \times \bar{E}-j \omega \mu \bar{H} .
\end{aligned}
$$

Note that these sources must equal zero in the solution system since the waveguide problem is source-free. Thus, the reaction [25] between arbitrary test fields $\left(\delta \bar{E}^{a}, \delta \bar{H}^{a}\right)$ and the trial sources $(\bar{J}, \bar{M})$ should vanish when the trial fields are equal to the true fields, i.e.,

$$
\delta I=\iint_{\Omega}\left(\delta \bar{E}^{a} \cdot \bar{J}-\delta \bar{H}^{a} \cdot \bar{M}\right) d \Omega=0
$$

where the integration region $\Omega$ extends over the whole space.

The equation (2) may be simplified to

$$
\begin{aligned}
\delta I & =0 \\
I & =\iint_{\Omega}\left(\bar{E}^{a} \cdot \bar{J}-\bar{H}^{a} \cdot \bar{M}\right) d \Omega
\end{aligned}
$$

if the variation is only partially operated on the test fields

0018-9480/88/0300-0576\$01.00 다요 IEEE 
$\left(\bar{E}^{a}, \bar{H}^{a}\right)$. However, too many unknowns are involved in (3); hence, some suitable constraints are added [24] to reduce the number of unknowns.

\section{SOlution Procedure}

\section{A. Formulation}

Consider the microstrip line shown in Fig. 1, for which we restrict the transverse components of the trial sources to be zero. This then leads to

$$
\left(\begin{array}{cc}
j \omega \epsilon & j \beta \hat{z} \times \\
j \beta \hat{z} \times & -j \omega \mu
\end{array}\right)\left(\begin{array}{l}
\bar{E}_{t} \\
\bar{H}_{t}
\end{array}\right)=\left(\begin{array}{cc}
0 & -\hat{z} \times \nabla_{t} \\
-\hat{z} \times \nabla_{t} & 0
\end{array}\right)\left(\begin{array}{l}
E_{z} \\
H_{z}
\end{array}\right)
$$

where $\left(\bar{E}_{t}, \bar{H}_{t}\right)$ are the trial transverse field components, and $\beta$ denotes the propagation constant.

By substituting (4) into (3) the $\left(E_{z}, H_{z}\right)$ variational formulation for the microstrip line can be obtained:

$$
\begin{gathered}
\delta\left(-j \omega \mu_{0} I\right)=\delta I_{0}=0 \\
I_{0}=\iint_{\Omega} d \Omega\left(\frac{1}{\epsilon_{r} \mu_{r}-\epsilon_{\mathrm{eff}}}\right)\left(\begin{array}{c}
\hat{z} \times \nabla_{t} E_{z}^{a} \\
\eta_{0} \nabla_{t} H_{z}^{a}
\end{array}\right)^{T} \\
\cdot\left(\begin{array}{cc}
\epsilon_{r} & -\sqrt{\epsilon_{\mathrm{eff}}} \\
\sqrt{\epsilon_{\mathrm{eff}}} & -\mu_{r}
\end{array}\right)\left(\begin{array}{c}
\hat{z} \times \nabla_{t} E_{z} \\
\eta_{0} \nabla_{t} H_{z}
\end{array}\right) \\
-k_{0}^{2} \iint_{\Omega} d \Omega\left(\begin{array}{c}
E_{z}^{a} \\
\eta_{0} H_{z}^{a}
\end{array}\right)^{T}\left(\begin{array}{cc}
\epsilon_{r} & 0 \\
0 & -\mu_{r}
\end{array}\right)\left(\begin{array}{c}
E_{z} \\
\eta_{0} H_{z}
\end{array}\right) .
\end{gathered}
$$

Here the integration region $\Omega$ extends over the whole $x-y$ plane, $k_{0}$ is the wavenumber in the air, $\eta_{0}$ is the intrinsic impedance of the air, $\mu_{0}$ is the permittivity of the air, and $\mu_{r}$ and $\epsilon_{r}$ denote the relative permittivity and permeability of the substrate, respectively. Also, the effective dielectric constant $\epsilon_{\text {eff }}$ is defined in terms of the propagation constant $\beta$ and $k_{0}$ as

$$
\epsilon_{\mathrm{eff}}=\left(\frac{\beta}{k_{0}}\right)^{2}=\left(\frac{\lambda_{0}}{\lambda_{g}}\right)^{2}
$$

where $\lambda_{0}$ and $\lambda_{g}$ are the wavelengths in the air and in the guide, respectively.

\section{B. Virtual Field in the Image Domain}

Let the space coordinate $(x, y)$ be mapped into the image $\left(x^{\prime}, y^{\prime}\right)$ through a certain conformal mapping. Then by the Cauchy-Riemann equation, the variational formulation (5) in the image coordinate can be reduced to

$$
\begin{aligned}
\delta I_{0}^{\prime}= & 0 \\
I_{0}^{\prime}= & \iint_{\Omega^{\prime}} d \Omega^{\prime}\left(\frac{1}{\epsilon_{r} \mu_{r}-\epsilon_{\mathrm{eff}}}\right)\left(\begin{array}{c}
\hat{z} \times \nabla_{t}^{\prime} E_{z}^{a} \\
\eta_{0} \nabla_{t}^{\prime} H_{z}^{a}
\end{array}\right)^{T} \\
& \cdot\left(\begin{array}{cc}
\epsilon_{r} & -\sqrt{\epsilon_{\mathrm{eff}}} \\
\sqrt{\epsilon_{\mathrm{eff}}} & -\mu_{r}
\end{array}\right)\left(\begin{array}{c}
\hat{z} \times \nabla_{t}^{\prime} E_{z} \\
\eta_{0} \nabla_{t}^{\prime} H_{z}
\end{array}\right) \\
& -k_{0}^{2} \iint_{\Omega^{\prime}} d \Omega^{\prime} J\left(\begin{array}{c}
E_{z}^{a} \\
\eta_{0} H_{z}^{a}
\end{array}\right)^{T}\left(\begin{array}{cc}
\epsilon_{r} & 0 \\
0 & -\mu_{r}
\end{array}\right)\left(\begin{array}{c}
E_{z} \\
\eta_{0} H_{z}
\end{array}\right)
\end{aligned}
$$

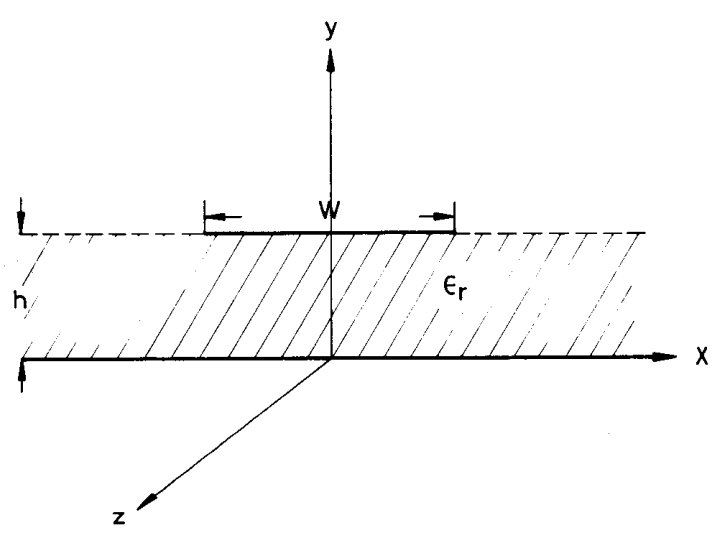

Fig. 1. Geometry of microstrip line.

Here and throughout this paper, the superscript ' denotes the corresponding variables or operators in the image domain. The integration region $\Omega^{\prime}$ is the image of the original region $\Omega$. Note that the forms of the functionals in (5) and (7) are almost identical, except that the second term must be multiplied by a Jacobian $J$ in the image domain.

The quantities $\left(\bar{E}_{t}^{\prime}, \bar{H}_{t}^{\prime}\right)$ in the image domain (named the virtual transverse fields) can be used to recover the transverse fields $\left(\bar{E}_{t}, \bar{H}_{t}\right)$ in the original space domain:

$$
\begin{aligned}
& \bar{E}_{t}=\frac{\left|\bar{E}_{t}^{\prime}\right|}{\sqrt{J}}\left[\cos \left(\Phi_{e}+\theta\right) \hat{x}+\sin \left(\Phi_{e}+\theta\right) \hat{y}\right] \\
& \bar{H}_{t}=\frac{\left|\bar{H}_{t}^{\prime}\right|}{\sqrt{J}}\left[\cos \left(\Phi_{h}+\theta\right) \hat{x}+\sin \left(\Phi_{h}+\theta\right) \hat{y}\right] \\
& \bar{E}_{t}^{\prime}=\left|\bar{E}_{t}^{\prime}\right|\left(\cos \Phi_{e} \hat{x}+\sin \Phi_{e} \hat{y}\right) \\
& \bar{H}_{t}^{\prime}=\left|\bar{H}_{t}^{\prime}\right|\left(\cos \Phi_{h} \hat{x}+\sin \Phi_{h} \hat{y}\right) .
\end{aligned}
$$

Here, $\Phi_{e}$ and $\Phi_{h}$ denote the angles between the two components of $\bar{E}_{t}^{\prime}$ and $\bar{H}_{t}^{\prime}$, respectively, while $\theta$ denotes the argument of the first derivative of the mapping function.

\section{Wheeler's Mapping}

We will show below that if Wheeler's mapping [7] is used for (7), the edge condition can be handled by the Jacobian $J$, and there will be no singularities in $\left(\bar{E}_{t}^{\prime}, \bar{H}_{t}^{\prime}\right)$. Wheeler's mapping $f\left(z^{\prime}\right)$ and its Jacobian are

$$
\begin{array}{r}
z=f\left(z^{\prime}\right)=\frac{h}{\pi}\left[j \pi+d \tanh \frac{z^{\prime}}{2}-z^{\prime}\right] \\
J\left(z^{\prime}\right)=\left|\frac{h}{\pi}\left[\frac{d}{\left(1+\cosh z^{\prime}\right)}-1\right]\right|^{2}
\end{array}
$$

where $z(=x+j y)$ and $z^{\prime}\left(=x^{\prime}+j y^{\prime}\right)$ are the complex numbers that represent the points of the original and image domains, respectively. In (12), $d$ is a parameter which depends on the height $h$ of the dielectric and the width $w$ of the strip. Do not confuse $z$ here with the spatial $z$ coordinate defined earlier. 


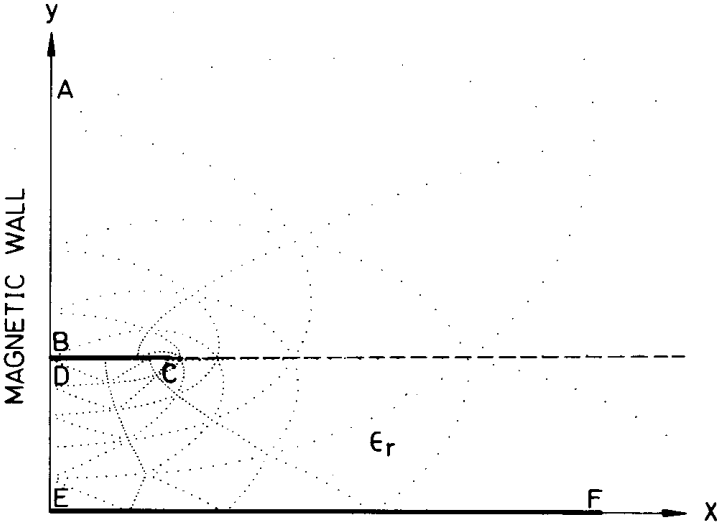

Fig. 2. Half structure and subdivisions in original $(x, y)$ domain.

The Jacobian around the strip edge is found to be

$$
J \rightarrow r \quad \text { near the edge }
$$

where $r$ is the distance from the edge in the original domain. Since the transverse fields near the strip edge would be proportional to $r^{-(1 / 2)}$, the values of the virtual transverse fields become smooth and finite. Hence, the transverse fields can be obtained indirectly without the problems of singularities by first calculating the virtual transverse fields, and then dividing them by the square root of the Jacobian.

\section{Finite Element Method}

The fields in the image domain can now be solved numerically through the finite element method. From the symmetry with respect to the $y-z$ plane shown in Fig. 1, we may place a magnetic wall at $x=0$ if only the lowest mode ( $E_{z}$ even and $H_{z}$ odd) is concerned. We now only need to consider the half structure shown in Fig. 2.

For convenience, the triangular elements in Fig. 3 are chosen in the image domain. In each element, the field $\psi$ which represents $E_{z}$ or $\eta_{0} H_{z}$ is written as

$$
\psi\left(x^{\prime}, y^{\prime}\right)=\sum_{i=1}^{6} \psi_{i} B_{i}(\zeta, \eta)
$$

where $\psi_{i}$ is the field value at node $i$ of the element, and $B_{i}$ is a quadratic interpolation basis function [26]. The integral over each element, furthermore, is approximated by the seven-point Gauss-Hammer quadratic formula [26].

After expanding $\psi$ in each element by (15), the matrix equation (16) can be obtained by the Rayleigh-Ritz procedure [24]:

$$
A \cdot \Psi=k_{0}^{2} B \cdot \Psi .
$$

Here $\Psi$ is the column vector corresponding to the nodal unknowns, and both $A$ and $B$ are known sparse matrices. Although $A$ is not positive definite, (16) can still be effectively solved by the determinant search together with the inverse iteration method [27].

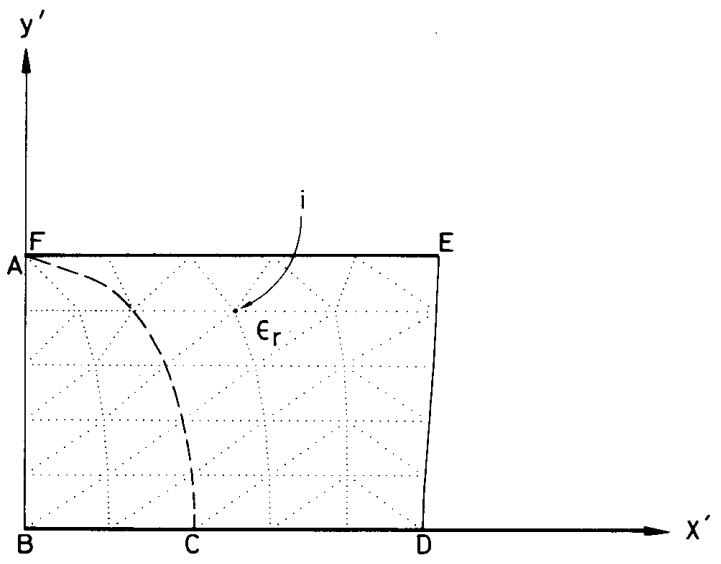

Fig. 3. Corresponding subdivisions in image $\left(x^{\prime}, y^{\prime}\right)$ domain (number of elements $=M_{x}^{\prime} \times M_{y}^{\prime}=5 \times 5=25$ ).

\section{RESUlts AND Discussions}

Based on the variational conformal mapping technique, a Fortran program has been implemented on the Vax$11 / 780$ computer. To check the validity and accuracy of this technique, the effective dielectric constants of several typical microstrip lines $\left(\epsilon_{r}=8\right.$ and $w=h=0.005 \lambda_{0} \sim$ $0.4 \lambda_{0}$ ) are investigated by choosing various discretizations for the mesh division. It is found that a moderate mesh division of $9 \times 9=81$ elements is usually enough to give results with four-digit accuracy.

Numerical results for the characteristics of the microstrip line, including the effective dielectric constant, the current distributions on the strip, and the characteristic impedance, will be presented and discussed in this section.

\section{A. Effective Dielectric Constant}

In Fig. 4, the effective dielectric constants for several structures are presented. Our computed results for the case with strip width $3.17 \mathrm{~mm}$ agree well with Itoh's [13] and Denlinger's [16] results, especially below $10 \mathrm{GHz}$. For higher frequency, Itoh's and Denlinger's results become inaccurate because their approaches have to assume the forms of the current distributions, and a rough guess of current distributions may result in error at higher frequency [28]. This point will be further verified by showing the current distributions in the next subsection. Our results should be more appropriate even at higher frequency, because we make minimal assumption for the current distributions.

Table III of [29] is reproduced here in Table I. On the whole, there exist discrepancies of less than 0.5 percent between our results and Kobayashi's. For several cases, the discrepancies may be up to 2 percent.

The relative wavelengths $\left(\lambda_{g} / \lambda_{0}\right)$ for several dielectric constants $\epsilon_{r}$ are shown in Fig. 5. Except that they are somewhat lower due to shielding, our results are in agreement with those of Itoh and the experimental data by Cohen [13]. 


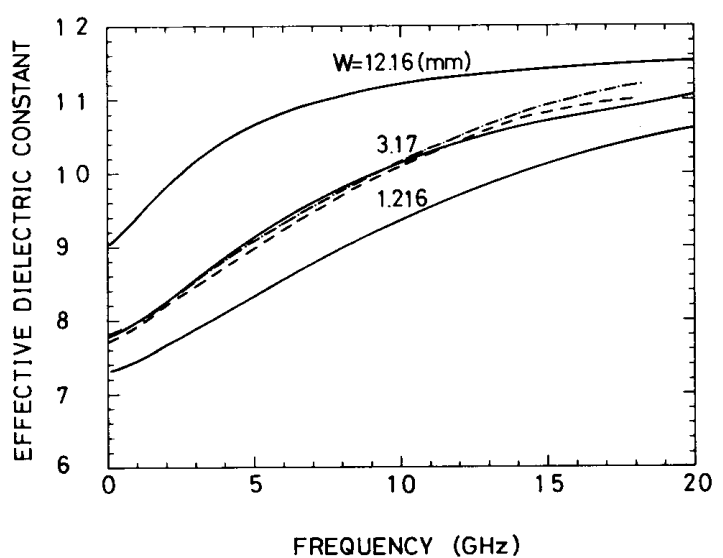

Fig. 4. Effective dielectric constant versus frequency $(h=3.04 \mathrm{~mm}$ $w=12.16,3.17,1.216 \mathrm{~mm}$, and $\epsilon_{r}=11.7$ ). Denliner [16] ---; Itoh [13] $-\cdot-\cdot-\cdot-$; present method

TABLE I

EFFECTIVE DIELECTRIC CONSTANT

\begin{tabular}{|c|c|c|c|c|c|c|c|c|c|c|}
\hline \multicolumn{2}{|c|}{$\epsilon_{r}{ }^{w / h} / \lambda_{0}$} & 0 & 0.005 & 0.05 & 0.1 & 0.2 & 0.3 & 0.4 & 0.7 & 1.0 \\
\hline 2 & $\begin{array}{c}0.4 \\
1 \\
2\end{array}$ & $\begin{array}{l}1.60424 \\
1.64721 \\
1.69845\end{array}$ & $\begin{array}{l}1.605 \\
1.648 \\
1.700\end{array}$ & $\begin{array}{l}1.632 \\
1.685 \\
1.748\end{array}$ & $\begin{array}{l}1.670 \\
1.730 \\
1.799\end{array}$ & $\begin{array}{l}1.743 \\
1.807 \\
1.869\end{array}$ & $\begin{array}{l}1.800 \\
1.859 \\
1.910\end{array}$ & $\begin{array}{l}1.846 \\
1.894 \\
1.995\end{array}$ & $\begin{array}{l}1.920 \\
1.947 \\
1.969\end{array}$ & $\begin{array}{l}1.956 \\
1.969 \\
1.980\end{array}$ \\
\hline 4 & $\begin{array}{c}0.4 \\
1 \\
2\end{array}$ & $\begin{array}{l}2.78971 \\
2.91690 \\
3.07157\end{array}$ & $\begin{array}{l}2.794 \\
2.924 \\
3.083\end{array}$ & $\begin{array}{l}2.933 \\
3.109 \\
3.319\end{array}$ & \begin{tabular}{|l|}
3.108 \\
3.319 \\
3.529
\end{tabular} & $\begin{array}{r}3.415 \\
3.599 \\
3.754\end{array}$ & $\begin{array}{l}3.614 \\
3.746 \\
3.852\end{array}$ & $\begin{array}{l}3.737 \\
3.830 \\
3.899\end{array}$ & \begin{tabular}{|r|}
3.896 \\
3.934 \\
3.948
\end{tabular} & $\begin{array}{r}3.944 \\
3.9556 \\
3.968\end{array}$ \\
\hline 8 & $\begin{array}{c}0.1 \\
0.4 \\
1 \\
2 \\
10\end{array}$ & $\begin{array}{l}5.02111 \\
5.14654 \\
5.44052 \\
5.80225 \\
6.88551\end{array}$ & $\begin{array}{l}5.033 \\
5.166 \\
5.471 \\
5.851 \\
6.986\end{array}$ & $\begin{array}{l}5.384 \\
5.657 \\
6.130 \\
6.647 \\
7.606\end{array}$ & $\begin{array}{l}5.863 \\
6.238 \\
6.753 \\
7.205 \\
7.786\end{array}$ & $\begin{array}{l}6.791 \\
7.066 \\
7.393 \\
7.650 \\
7.925\end{array}$ & $\begin{array}{l}7.365 \\
7.472 \\
7.654 \\
7.766 \\
7.963\end{array}$ & $\begin{array}{l}7.617 \\
7.671 \\
7.778 \\
7.843 \\
7.979\end{array}$ & $\begin{array}{l}7.872 \\
7.881 \\
7.914 \\
7.928 \\
7.993\end{array}$ & $\begin{array}{l}7.938 \\
7.939 \\
7.948 \\
7.955 \\
7.996\end{array}$ \\
\hline 16 & $\begin{array}{c}0.4 \\
1 \\
2\end{array}$ & $\begin{array}{l}9.86047 \\
10.4786 \\
11.2545\end{array}$ & $\begin{array}{l}9.929 \\
10.61 \\
11.44\end{array}$ & $\begin{array}{l}11.48 \\
12.61 \\
13.71\end{array}$ & \begin{tabular}{|l|}
13.10 \\
14.08 \\
14.86
\end{tabular} & $\begin{array}{l}14.79 \\
15.21 \\
15.45\end{array}$ & $\begin{array}{l}15.43 \\
15.59 \\
15.69\end{array}$ & \begin{tabular}{|l|}
15.64 \\
15.75 \\
15.79
\end{tabular} & $\begin{array}{l}15.88 \\
15.89 \\
15.92\end{array}$ & $\begin{array}{l}15.94 \\
15.95 \\
15.96\end{array}$ \\
\hline 128 & $\begin{array}{c}0.4 \\
1 \\
2\end{array}$ & $\begin{array}{l}75.6999 \\
80.9649 \\
87.5397\end{array}$ & $\begin{array}{l}78.66 \\
85.28 \\
93.64\end{array}$ & $\begin{array}{l}111.4 \\
117.4 \\
120.4\end{array}$ & \begin{tabular}{|l|}
122.4 \\
124.2 \\
125.2
\end{tabular} & $\begin{array}{l}126.5 \\
126.7 \\
127.0\end{array}$ & $\begin{array}{l}127.3 \\
127.4 \\
127.5\end{array}$ & $\begin{array}{l}127.6 \\
127.7 \\
127.7\end{array}$ & $\begin{array}{l}127.9 \\
127.9 \\
127.9\end{array}$ & $\begin{array}{l}127.9 \\
127.9 \\
127.9\end{array}$ \\
\hline & & 0 & $10^{-7}$ & $10^{-6}$ & $10^{-6}$ & $10^{-4}$ & 0.001 & 0.002 & 0.003 & 0.004 \\
\hline
\end{tabular}

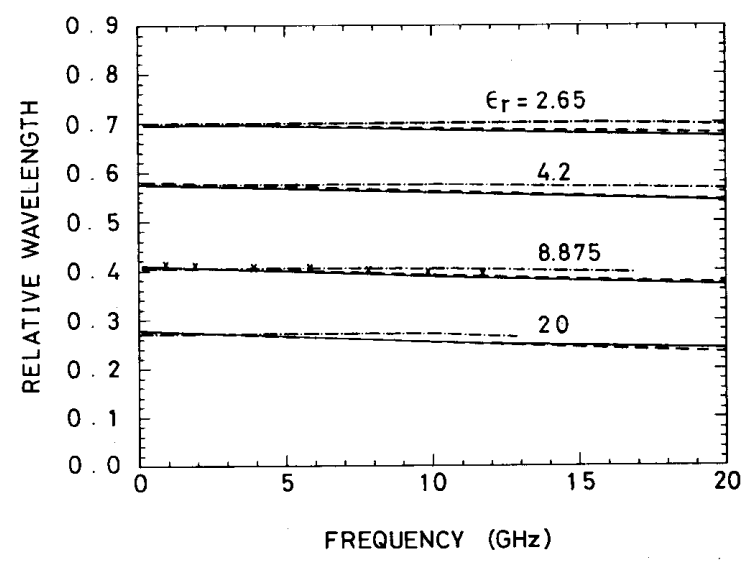

Fig. 5. Relative wavelength versus frequency $(h=1.27 \mathrm{~mm}, w=1.27$ $\mathrm{mm}$, and $\left.\epsilon_{r}=2.65,4.2,8.875,20\right)$. Itoh [13] ----; Bhartia [18] -...--; experiment by Cohen [13] $\times \times \times$; present method - .

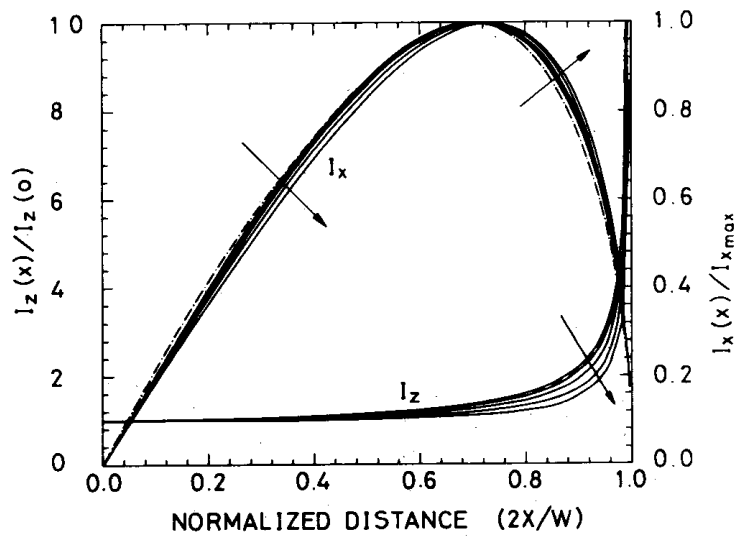

Fig. 6. Normalized current distributions versus normalized distance with frequencies as parameters $\left(h=3.04 \mathrm{~mm}, w=3.17 \mathrm{~mm}, \epsilon_{r}=11.7\right.$, and frequency $=1,3,6,10,15,20 \mathrm{GHz}$ ). Kuester and Chang [21] ----; Kobayashi [20] -.-.--; present method - The arrows indicate the increase of frequencies.

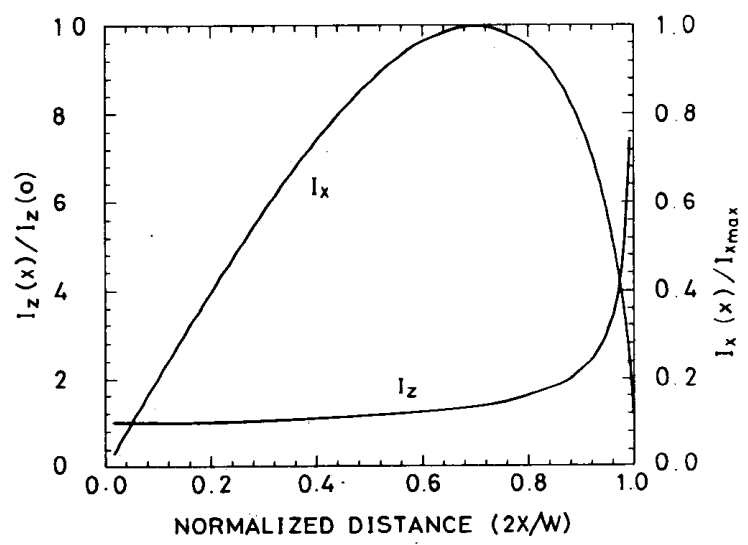

Fig. 7. Normalized current distributions versus normalized distance with dielectric constants as parameters $(h=1.27 \mathrm{~mm}, w=1.27 \mathrm{~mm}$, $\epsilon_{r}=4.2,8.875,20$, and frequency $=1 \mathrm{GHz}$ ).

\section{B. Current Distributions}

It has been shown that the edge condition can be handled by Wheeler's mapping in our method. As shown in Fig. 6, the current distributions $\left(I_{z}(x) / I_{z}(0)\right)$ and $\left(I_{x}(x) / I_{x}\right)$ at lower frequency agree well with the solutions by the Green function's technique together with quasi-static approximation [20], [21]. For higher frequency, more energy is confined in the center region, which makes more current flow through the strip center part. Meanwhile, less current gathers around the strip edge and hence a steeper slope of current distribution is observed near the edge. Obviously, this effect cannot be reflected by the quasi-static results [20], [21], which are independent of frequency.

Next, the longitudinal and transverse current distributions for several values of dielectric constants $\left(\epsilon_{r}=4.2\right.$, $8.875,20$ ) at $1 \mathrm{GHz}$ are presented in Fig. 7. Like Kobayashi's results [20] and Denlinger's approximate rela- 


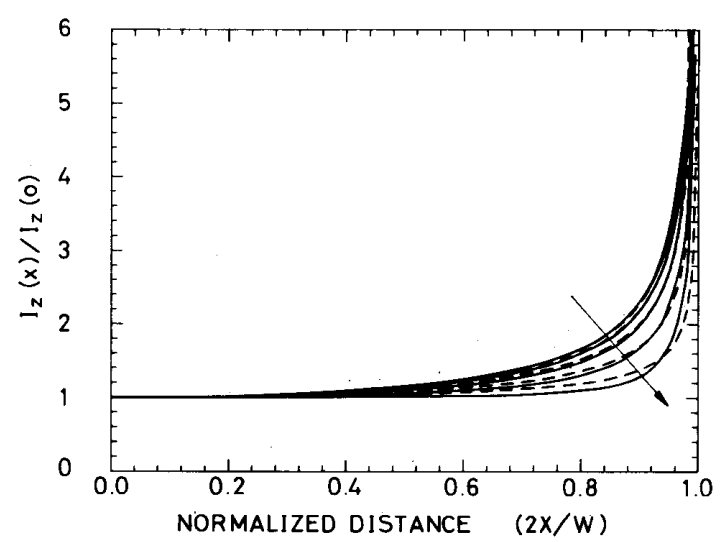

Fig. 8. Normalized current distributions versus normalized distance with strip width $w$ as parameters $(h=3.04 \mathrm{~mm}, w=0.304,1.216,3.17$, $6.08,12.16,30.4 \mathrm{~mm}, \epsilon_{r}=11.7$, and frequency $=1 \mathrm{GHz}$ ). Kobayashi $[20]----$; present method - . The arrow indicates increasing $w / h$ values.

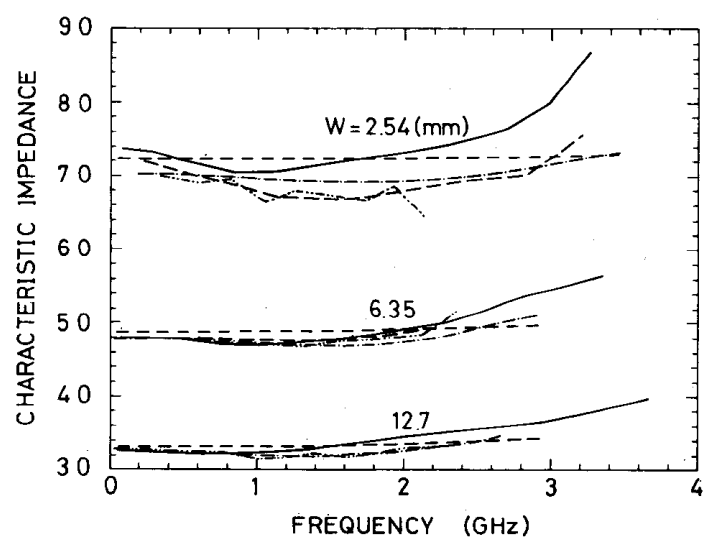

Fig. 9. Characteristic impedance versus frequency $(h=6.35 \mathrm{~mm}, w=$ $2.54,6.35,12.7 \mathrm{~mm}$, and $\left.\epsilon_{r}=9.74\right)$. Getsinger [30] ----; Bhartia [18]

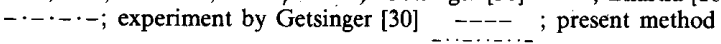

tion [16], they are hardly distinguished, i.e., the current distributions are nearly independent of dielectric constants. The current distributions for different $w / h$ values are presented in Fig. 8. On the whole, our results at lower frequency are in agreement with Kobayashi's closed forms [20].

\section{Characteristic Impedance}

The characteristic impedance $Z_{0}$ suggested by Gestinger [30] can be evaluated either in the original domain or in the image domain:

$Z_{0}=\frac{2 P}{\left|I_{z t}\right|^{2}}=\frac{2 \iint_{\Omega} \bar{E}_{t} \times \bar{H}_{t}^{*} \cdot \hat{z} d \Omega}{\left|\int_{l} \hat{n} \times \bar{H}_{t} \cdot \hat{z} d l\right|^{2}}=\frac{2 \iint_{\Omega^{\prime}} \bar{E}_{t}^{\prime} \times \bar{H}_{t}^{\prime *} \cdot \hat{z} d \Omega^{\prime}}{\left|\int_{l^{\prime}} \hat{n} \times \bar{H}_{t}^{\prime} \cdot \hat{z} d l^{\prime}\right|^{2}}$ where $P$ and $I_{z t}$ denote the total power and the total current on the strip in the propagation direction. Obviously, if the image-domain expression is used, the integration covers only a finite region, and will include no singularities. Thus, we compute the characteristic impedance on the image domain.

In Fig. 9, our results are compared with those of various methods and some experimental data with shielding [18], [30]. At lower frequency, our results are in agreement with the experimental data. However, some numerical variations within 3 percent may appear in our computed values, even with rather dedicated mesh divisions.

\section{Conclusions}

A new full-wave analysis of microstrip lines has been presented. The method is characterized by its superiority in handling the edge condition without resorting to any presumed current distribution. Numerical results for the effective dielectric constant, the current distributions, and the characteristic impedance have also been shown and compared with available literature. Our results reveal the frequency dependence of the current distributions, and are more reasonable at higher frequency.

It should be emphasized that this approach is very flexible: It can be applied directly to microstrip lines with inhomogeneous and even anisotropic substrates. The effect of strip thickness can also be treated through adopting the Schwartz-Christoffel transformation instead of Wheeler's mapping. Further investigation is in progress and will appear in the near future.

\section{ACKNOWLEDGMENT}

The authors wish to express their deep appreciation to the members of the EM group at the National Taiwan University for valuable discussions.

\section{REFERENCES}

[1] J. Frey and K. Bhasin, Microwave Integrated Circuits. Dedham, MA: Artech House, 1985.

[2] K. C. Gupta, R. Garg, and I. J. Bahl, Microstrip Lines and Slotlines. Dedham, MA: Artech House, 1981.

[3] T. C. Edwards, Foundations for Microstrip Circuit Design. New York: Wiley, 1981.

[4] P. J. Meier, "Integrated fin-line millimeter components," IEEE Trans. Microwave Theory Tech., vol. MTT-22, pp. 1209-1216, 1974

[5] M. V. Schneider, "Microstrip lines for microwave integrated circuits," Bell Syst. Tech. J., vol. 48, pp. 1421-1444, 1969.

[6] E. Yamashita and R. Mittra, "Variational method for the analysis of microstrip lines," IEEE Trans. Microwave Theory Tech., vol. MTT-16, pp. 251-256, 1968.

[7] H. A. Wheeler, "Transmission line properties of parallel strips separated by a dielectric sheet," IEEE Trans. Microwave Theory Tech., vol. MTT-13, pp. 172-185, 1965.

[8] H. A. Wheeler, "Transmission line properties of parallel wide strips by conformal mapping approximation," IEEE Trans. Microwave Theory Tech., vol. MTT-12, pp. 280-289, 1964.

[9] M. Hashimoto, "A riogorous solution for dispersive microstrip," IEEE Trans. Microwave Theory Tech., vol. MTT-33, pp. 1131-1137, 1985.

[10] E. F. Kuester and D. C. Chang, "Theory of dispersion in microstrip of arbitrary width," IEEE Trans. Microwave Theory Tech., vol. MTT-28, pp. 259-265, 1980.

[11] A. Farrar and A. T. Adams, "Computation of propagation constants for the fundamental and higher order modes in microstrip," 
IEEE Trans. Microwave Theory Tech., vol. MTT-24, pp. 456-460, 1976.

[12] T. Itoh and R. Mittra, "A technique for computing dispersion characteristics of shielded microstrip lines," IEEE Trans. Microwave Theory Tech., vol. MTT-22, pp. 896-898, 1974

[13] T. Itoh and R. Mittra, "Spectral-domain approach for calculating the dispersion characteristics of microstrip lines," IEEE Trans. Microwave Theory Tech., vol. MTT-21, p. 496-498, 1973.

[14] M. K. Krage and G. I. Haddad, "Frequency-dependent characteristics of microstrip transmission lines," IEEE Trans. Microwave Theory Tech., vol. MTT-20, pp. 678-688, 1972.

[15] R. Mittra and T. Itoh, "A new technique for the analysis of the dispersion characteristics of microstrip lines," IEEE Trans. Microwave Theory Tech., vol. MTT-19, pp. 47-56, 1971.

[16] E. J. Denlinger, "A frequency dependent solution for microstrip transmission lines," IEEE Trans. Microwave Theory Tech., vol. MTT-19, pp. 30-39, 1971.

[17] P. Daly, "Hybrid-mode analysis of microstrip by finite-elemen methods," IEEE Trans. Microwave Theory Tech., vol. MTT-19, p. 19-25, 1971.

[18] P. Bhartia and P. Pramanick, "A new microstrip dispersion model," IEEE Trans. Microwave Theory Tech., vol. MTT-32, pp. 1379-1384, 1984.

[19] W. J. Getsinger, “Microstrip dispersion model," IEEE Trans. Microwave Theory Tech., vol. MTT-21, pp. 34-39, 1973.

[20] M. Kobayashi, "Longitudinal and transverse current distributions on microstriplines and their closed-form expression," IEEE Trans. Microwave Theory Tech., vol. MTT-33, pp. 784-788, 1985.

[21] E. F. Kuester and D. C. Chang, "Close-form expressions for the current or charge distribution on parallel strips or microstrips," IEEE Trans. Microwave Theory Tech., vol. MTT-28, pp. 254-259, 1980.

[22] M. Kobayashi, "Analysis of the microstrip and the electrooptic light modulators," IEEE Trans. Microwave Theory Tech., vol. MTT-26, pp. 119-126, 1978 .

[23] R. B. Wu and C. H. Chen, "A variational analysis of dielectric waveguides by the conformal mapping technique," IEEE Trans. Microwave Theory Tech., vol. MTT-33, pp. 681-685, 1985.

[24] R. B. Wu and C. H. Chen, "On the variational reaction theory for dielectric waveguides," IEEE Trans. Microwave Theory Tech., vol. MTT-33, pp. 477-483, 1985.

[25] V. H. Rumsey, "Reaction concept in electromagnetic theory," Phys. Rev., vol. 94, pp. 1483-1491, 1954

[26] S. S. Rao, The Finite Element Method in Engineering. New York: Maxwell House, 1982.

[27] K. J. Bathe and E. L. Wilson, Numerical Methods in Finite Element Analysis. Englewood Cliffs, NJ: Prentice-Hall, 1976.

[28] E. F. Kuester and D. C. Chang, "An appraisal of methods for computation of the dispersion characteristics of open microstrip," IEEE Trans. Microwave Theory Tech., vo.. MTT-27 pp. 691-694, 1979.

[29] M. Kobayashi and F. Ando, "Dispersion characteristics of open microstrip lines," IEEE Trans. Microwave Theory Tech., vol. MTT35 , pp. 101-105, 1987.

[30] W. J. Getsinger, "Measurement and modeling of apparent characteristic impedance of microstrip," IEEE Trans. Microwave The ory Tech., vol. MTT-31, pp. 624-632, 1983.

\section{功}

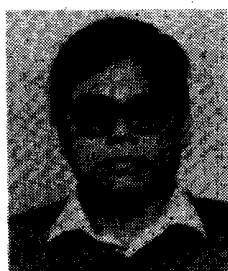

Chin Shih was born in Tainan, Taiwan, Republic of China, on January 26,1961. He received the B.S. degree from the Department of Communication Engineering, National Chiao Tung University, Hsinchu, Taiwan, in 1984. Currently, he is completing the M.S. degree at the Department of Electrical Engineering, National Taiwan University, Taipei, Taiwan, where he concentrates on the study of the microstrip lines.

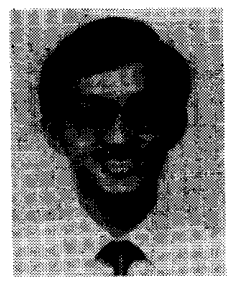

Ruey-Beei Wu was born in Tainan, Taiwan, Republic of China, on October 27, 1957. He received the B.S.E.E. degree from National Taiwan University, Taipei, Taiwan, in 1979, and the $\mathrm{Ph} . \mathrm{D}$. degree from the same university in 1985 .

In 1982, he joined the faculty of the Department of Electrical Engineering, National Taiwan University, where he is now an Associate Professor. In 1986, he was a Visiting Scientist for one year at the IBM General Technology Division Laboratory, East Fishkill Facility, Hopewell Junction, NY. He is involved in numerical techniques for electromagnetics and electrical analysis for computer packaging.

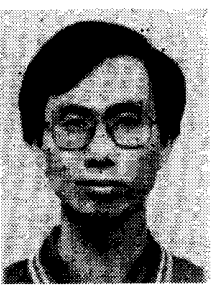

Shyh-Kang Jeng (M'87) was born in I-Lan, Taiwan, Republic of China, on May 6, 1957. He received the B.S.E.E. and the $\mathrm{Ph}$.D. degrees from National Taiwan University in 1979 and 1983, respectively.

In 1981 he joined the faculty of the Department of Electrical Engineering, National Taiwan University, where he is now an Associate Professor. From 1984 to 1985 he was an electronic data processing officer and an instructor on information system analysis and design at the National Defense Management College, Chung-Ho, Taiwan, R O.C. From September 1985 to August 1986 he was a Visiting Research Associate Professor at the University of Illinois, Urbana. His current interest is in applying numerical techniques, computer graphics, and computer algebra to EM field problems.

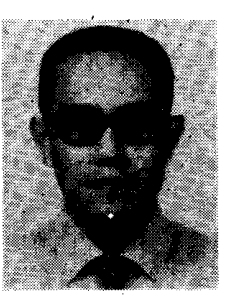

Chun Hsiung Chen was born in Taipei, Taiwan, Republic of China, on March 7, 1937. He received the B.S.E.E. degree from National Taiwan University, Taipei, Taiwan, in 1960, the M.S.E.E. degree from National Chiao Tung University, Hsinchu, Taiwan, in 1962, and the Ph.D. degree in. electrical engineering from National Taiwan University in 1972 .

In 1963 he joined the faculty of the Department of Electrical Engineering, National Taiwan University, where he is now a Professor. From August 1982 to July 1985 he was Chairman of the department. In 1974 he was a Visiting Researcher for one year at the Department of Electrical Engineering and Computer Sciences, University of California, Berkeley. From August 1986 to July 1987, he was a Visiting Professor at the Department of Electrical Engineering, University of Houston, TX. His areas of interest include antenna and waveguide analysis, propagation and scattering of waves, and numerical techniques in electromagnetics. 